\title{
Electrochemical Studies of Carbonated Hydroxyapatite/CHS Nanocomposite $\uparrow$
}

\author{
C. P. DHANALAKSHMI ${ }^{\mathrm{a}}$, L. VIJAYALAKSHMI ${ }^{\mathrm{b}}$, \\ R. SURESH ${ }^{\mathrm{a}}, \mathrm{K}$. GIRIBABU ${ }^{\mathrm{a}}$ and V. NARAYANAN ${ }^{\mathrm{a} *}$
}

${ }^{a}$ Department of Inorganic Chemistry, University of Madras, Guindy Maraimalai Campus, Chennai-600025, Tamilnadu, India

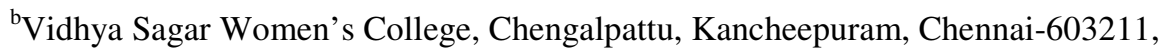

Tamilnadu, India

vnnara@yahoo.co.in

Received 24 January 2013 / Accepted 15 February 2013

\begin{abstract}
Carbonated hydroxyapatite /CHS nanocomposites of varying composition for biomaterial applications have been synthesized. The Carbonated hydroxyapatite /CHS nanocomposite materials were characterized by XRD, FTIR, FESEM, EDAX, HRTEM and XPS. Carbonated hydroxyapatite (CHAp) nanorod embedded composite was prepared using chitosan (CHS) as a matrix with different weight percentages (wt \%). The results indicated that the size and crystallinity of CHAp nano particles decreases with increase in CHS concentration in the composite. FESEM confirms the presence of CHAp nanorod crystals in CHS matrix. Cyclic voltammograms (CVs) were recorded to evaluate the electrocatalytic behaviors of the modified electrode towards the oxidation of 4-NP in the potential range of 0 to $+1.0 \mathrm{~V}$. A glassy carbon electrode was modified with carbonated hydroxyapatite nanopowder (CHAp-NP) and characterized in terms of electrochemical oxidation of 4-nitrophenol (4-NP) via cyclic voltammetry. The catalytic reaction facilitates electron transfer between 4-NP and the modified electrode, as a result the electrochemical oxidation of 4-NP becomes easier. The reason for this is that the CHAp-CHS 80 can act as a promoter to increase the rate of electron transfer, lower the overpotential of 4-NP at the bare electrode, and the anodic peak shifts less positive potential. Thus, it is clear that CHAp-CHS 80 modified GCE can be successfully used for the determination of organic pollutant.
\end{abstract}

Keywords: Carbonated hydroxyapatite, Electrochemical oxidation, Nanocrystalline, Chitosan

\section{Introduction}

A critical challenge in the design of these hybrid inorganic-organic systems is a control of the mixing between the two dissimilar phases. A composite material is a macroscopic combination of two or more distinct materials, having a recognizable interface between them. Composites are used not only for their structural properties, but also for electrical, thermal, tribological and environmental applications. Modern composite materials are usually optimized to achieve a particular balance of properties for a given range of applications. 
4-NP is widely used as intermediates in the production of pharmaceuticals, pesticides and dyestuffs, such as parathion insecticide which can also reversely hydrolyze to form 4-NP. In addition, 4-NP can also be used as leather fungicide and acid-base indicator. Therefore, 4-NP will be inevitably released into environment to cause pollution in the process of fabrication and application. Based on the above description, it is important to develop simple and reliable method for determination of trace amounts of 4-NP in environments ${ }^{1,2}$. Up to now, various techniques have been investigated to determine 4-NP, such as spectrophotometry, fluorescence, high-performance liquid chromatography ${ }^{3}$, liquid chromatography with electrochemical detection, capillary zone electrophoresis ${ }^{4}$, fiber optode ${ }^{5}$ and electrochemical method $^{6}$. Among them, electrochemical methods have received considerable attention because of the advantage of fast response, cheap instrument, low cost, simple operation, timesaving, high sensitivity and selectivity, real-time detection in in-situ condition. However, until now, many electrochemical determination techniques mainly focus on utilizing the reduction of nitryl in 4-NP, not the oxidation of hydroxide radical. Thus, the determination would be interfered by the oxygen molecule dissolved in solvent. In order to eliminate it, purging nitrogen for a certain time was employed. But this will make the determination more complicated and time-consuming. Moreover, the reduction of nitryl is more complicated than the oxidation of hydroxide radical. Therefore, electrochemical determination of 4-NP using the oxidation signal should be an appropriate alternative. Nevertheless, the use of bare electrode has revealed the drawback of weak electrochemical response and low sensitivity. Consequently, chemically modified electrodes (CMEs) have been widely investigated with various modification materials, especially nanophase materials, such as $\mathrm{MWCNT}^{7}, \mathrm{SWCNT}^{8}$, gold nanoparticles ${ }^{9}$, $\mathrm{TiO}_{2},{ }^{10}$ zeolite $^{11}$, sodium montmorillonite ${ }^{12}$ and $\mathrm{HAp}^{13,14}$. Among them, nano HAp has advantages of abundant in nature, cheap, readily available and stable in water and nontoxicity. Due to the high surface area and strong adsorption ability, a film of silica/HRP-HAp has been deposited on GCE surface to investigate the direct electron transfer of immobilized $\mathrm{HRP}$ and $\mathrm{H}_{2} \mathrm{O}_{2}$ determination $^{15}$. El Mohammedi et al. have evaluated the analytical performance of nano HApCPE for the detection of trace lead (II), paraquat and 4-NP. They found that HAp can effectively improve the electrochemical response due to its excellent adsorption property ${ }^{16,17}$. So, nano HAp should be a suitable electrode modified material for pollution determination.

The aim of the present work is to develop a simple, reliable and sensitive electrochemical method for the determination of 4-NP(4-nitrophenol) based on the unusual properties of polymer composites of nano CHAp-CHS -NP modified electrode. The modified electrode showed good electrochemical oxidation to 4-NP with an increase of the oxidation peak current. The electrocatalytic determination of nitrophenols were carried out using the polymer nanocomposite modified electrodes and found to be good sensors for the detection of nitro organic compounds.

\section{Experimental}

The raw materials required to start the processing of the composite were: analytical grade calcium hydroxide $\left(\mathrm{Ca}\left(\mathrm{NO}_{3}\right)_{2}\right),\left(\mathrm{NH}_{4}\right)_{2} \mathrm{HPO}_{4}$ and $\left(\mathrm{NH}_{4}\right)_{2} \mathrm{CO}_{3}$ obtained from Merck (India) and PEG (MW 6000) purchased from Loba (India). Doubly distilled water was used as the solvent.

\section{Methods}

\section{Synthesis of nano CHAp}

The nano CHAp was synthesized by following a modified wet chemical method. $\mathrm{Ca}\left(\mathrm{NO}_{3}\right)_{2}$ and PEG were dissolved in $50 \mathrm{~mL}$ distilled water to form a $\mathrm{Ca}\left(\mathrm{NO}_{3}\right)_{2}$ solution with a 
PEG:Ca ${ }^{2+}$ weight ratio of 4:1. $\left(\mathrm{NH}_{4}\right)_{2} \mathrm{HPO}_{4}$ and $\left(\mathrm{NH}_{4}\right)_{2} \mathrm{CO}_{3}$ were first dissolved in $50 \mathrm{~mL}$ distilled water to form a clear $0.10 \mathrm{M}$ phosphate solution with an initial molar ratio of $\mathrm{CO}_{3} / \mathrm{PO}_{4}$ of $1: 1$ and then added drop wise to the polymer-Ca( $\left(\mathrm{NO}_{3}\right)_{2}$ solution with an initial $\mathrm{Ca} / \mathrm{P}$ molar ratio of 1.60 ; the precipitation reaction was maintained at $5{ }^{\circ} \mathrm{C}$ under vigorous stirring for $30 \mathrm{~min}$. The powder sample of CHAp was obtained after heat treating the precipitate at $800^{\circ} \mathrm{C}$ for $3 \mathrm{~h}$ in a furnace filled with air.

\section{Synthesis of CHAp-CHS nanocomposites}

The CHAp-CHS nanocomposite was synthesized by freeze gelation method. Water was used as the solvent to prepare polymer solution. CHS was dissolved by using magnetic stirrer for $3 \mathrm{~h}$ and the polymer solution was left overnight in room temperature to remove the air bubbles trapped in the viscous solution. Then suitable amount of CHAp was dispersed in deionised water by $30 \mathrm{~min}$ ultrasonication. Ultrasonication was necessary to avoid agglomeration of ceramic powder and to achieve proper dispersion. CHAp in water was mixed with polymer solution under agitation. The homogeneously mixed solution is immediately taken to deep freeze at $-18{ }^{\circ} \mathrm{C}$. After $48 \mathrm{~h}$ freezing the samples were freeze dried. The CHAp-CHS nano composites were coded as CHAp-CHS 20 to CHAp-CHS 80 where number denotes the wt $\%$ of CHS.

\section{Physical-chemical characterization}

The prepared samples were studied by Fourier Transform Infrared Spectroscopy (FTIR) using a Schimadzu FT-IR 300 series instrument (Shimadzu Scientific Instruments, USA). The FTIR spectra were obtained over the region $450-4000 \mathrm{~cm}^{-1}$ in pellet form for $1 \mathrm{mg}$ powder samples mixed with $200 \mathrm{mg}$ spectroscopic grade $\mathrm{KBr}\left(\right.$ ). Spectra were recorded at $4 \mathrm{~cm}^{-1}$ resolution averaging 80 scans. The structure of the samples were analyzed by X-Ray Diffraction (XRD) using a Rich Siefert 3000 diffractometer (Seifert, Germany) with $\mathrm{Cu}-\mathrm{K} \alpha_{1}$ radiation $(\lambda=1.5418$ $\AA ̊$ ). In this work a JEOL FX 2000 Transmission Electron Microscope and FEI Teccani F30 High Resolution Transmission Electron Microscope were used to study the morphology of the nanoparticles with a resolution of $5 \AA-10 \AA$. The powder samples were dispersed in Gatan G1 epoxy resin and dried at $353 \mathrm{~K}$ to get a hard and thick disc. Then thick discs were sliced to small pieces and mechanically polished to $\sim 0.15 \mu \mathrm{m}$.

\section{Results and Discussion Characterization of CHAp-CHS nanocomposites FT-IR analysis}

The FT-IR spectra of pure nano CHAp and CHAp-CHS nanocomposites are shown in Figure 1 The $v_{2}$ phosphate stretching mode is appeared at $527 \mathrm{~cm}^{-1}$ corresponds to $\mathrm{PO}_{4}^{3-}$ group in CHAp. The bands appeared at $1073-1114 \mathrm{~cm}^{-1}$ and $527-585 \mathrm{~cm}^{-1}$ corresponds to different modes of the $\mathrm{PO}_{4}{ }^{3-}$ group in carbonated hydroxyapatite. The observed bands at 602 $\mathrm{cm}^{-1}$ corresponds to O-P-O bending and $v_{1}$ symmetric P-O stretching modes. The $v_{1}$ symmetric stretching mode of phosphate group is observed at $955-962 \mathrm{~cm}^{-1}$. The observed bands at $1384-1466$ and $805-872 \mathrm{~cm}^{-1}$ are assigned to carbonate ions. The peak appeared at $1633-1649 \mathrm{~cm}^{-1}$ (amide I) with a minor shoulder at $1564 \mathrm{~cm}^{-1}$ (amide II) are attributed to acetylated amino and confirms that sample is not fully deacetylated. The peak at $1384 \mathrm{~cm}^{-1}$ was assigned to $\mathrm{C}-\mathrm{O}$ stretching of primary alcoholic group $\left(-\mathrm{CH}_{2} \mathrm{OH}\right)$ and the peak at 900 $\mathrm{cm}^{-1}$ is attributed to the $\mathrm{C}-\mathrm{O}$ stretching. The bands at $1550-1700 \mathrm{~cm}^{-1}$ are attributable to mode super position of the hydroxyapatite $\mathrm{OH}^{-}$group and the CHS amide I and amide II groups $^{17-20}$. A broad band appears from 3433-3436 and $2919 \mathrm{~cm}^{-1}$ corresponding to the broad $-\mathrm{OH}$ stretching bands between $3100-3450 \mathrm{~cm}^{-1}$ and the aliphatic $\mathrm{C}-\mathrm{H}$ stretching bands between $2918-2925 \mathrm{~cm}^{-1}$. 


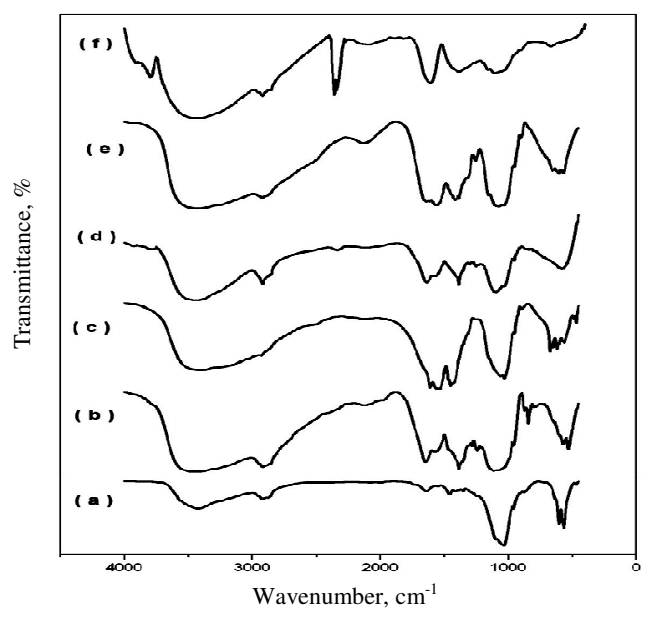

Figure 1. FTIR Spectra of (a) nano CHAp, (b) CHAp-CHS 20, (c) CHAp-CHS 40, (d) CHAp-CHS 60, (e) CHAp-CHS 80 and (f) CHS

\section{$X$-ray diffraction analysis}

The XRD patterns of nano CHAp and CHAp-CHS nanocomposites were taken. The patterns indicate the presence of amorphous CHAp. The broad peaks reveal that the particles sizes are very small in the range of 33 to $34 \mathrm{~nm}$. The reflection planes corresponding to the characteristic XRD spectral peaks of pure nano CHAp and CHAp-CHS nanocomposites are shown in Figure 2. The observed diffraction peaks are identified by standard JCPDS file (File No. 35-0180) and are assigned as crystalline CHAp. The XRD patterns show diffraction peaks with line broadening and high intensities, which confirms the nano size with crystalline nature. The crystallite size of the pure nano CHAp and CHAp-CHS nanocomposite is calculated by using Scherrer's formula. Figure 2 reveals that the crystallite size of nano CHAp decreases with increase in the composition of CHS.

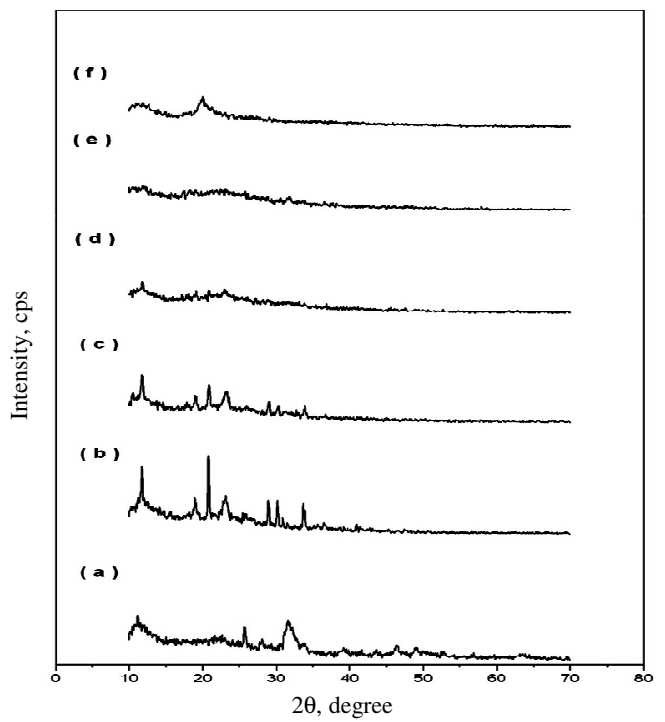

Figure 2. XRD Pattern of (a) nano CHAp, (b) CHAp-CHS 20, (c) CHAp-CHS 40, (d) CHAp-CHS 60, (e) CHAp-CHS 80 and (f) CHS 


\section{FESEM and EDAX analysis}

FESEM images of pure nano CHAp and different weight percentages of CHS compositions are illustrated in Figure 3. The FESEM picture shows that particles exhibit nanorod morphology. The particle size of pure nano CHAp is 25-70 nm. In case of composites, when the composition of CHS is added to CHAp, the rod-like morphology starts to disappear. The increase in the CHS compositions i.e., 20, 40, $60 \mathrm{wt}$. \% leads to a corresponding change from rod-like to an irregular morphology. Further, it is evident that the particle size decreases with increase in CHS composition. The elemental analysis (EDAX) of CHApCHS 40 nanocomposite is illustrated in Figure 3a. Mineral composition (calcium phosphate: $\mathrm{Ca}, \mathrm{O}, \mathrm{P})$ and organic content $(\mathrm{C}$ present in the nanocomposite CHAP-CHS is tested.
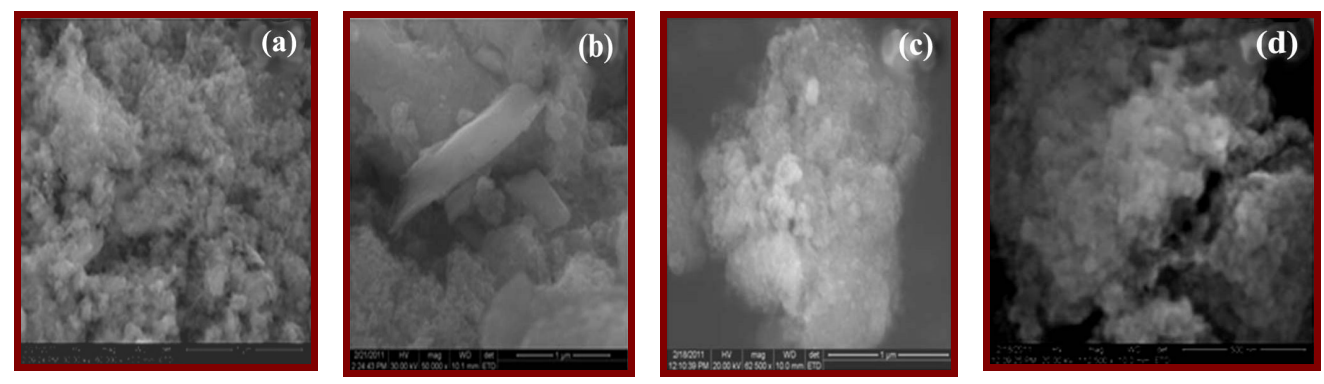

Figure 3. FE-SEM images of (a) nano CHAp, (b) CHAp-CHS 20, (c) CHAp-CHS 40 and (d) CHAp-CHS 60

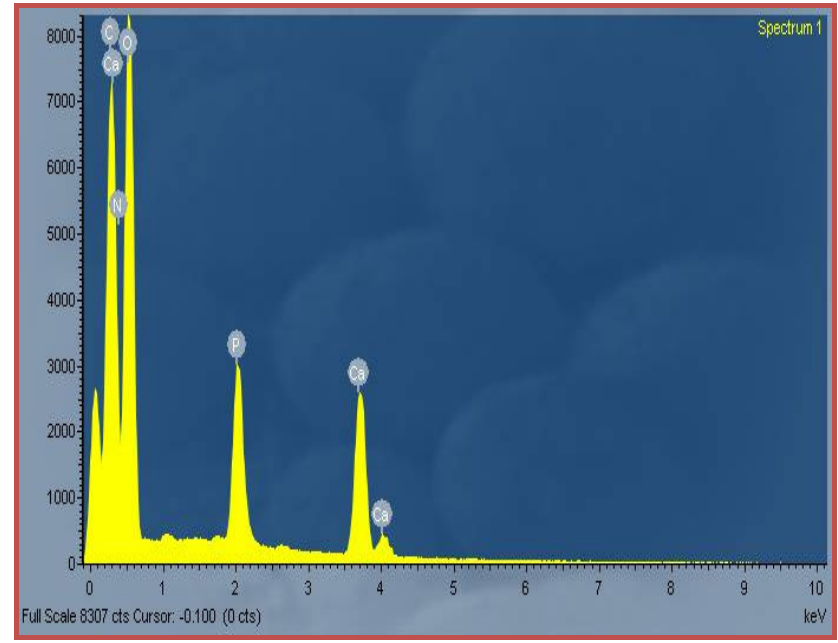

Figure 3a. EDAX spectrum of CHAp-CHS 40 nanocomposite

\section{HR TEM analysis}

Figure 4 shows the HRTEM photograph of CHAp-CHS 20 nanocomposite powder. The CHApCHS 20 nanocomposite particles shown in Figure 4 are in nanometer scale. This core/shell nanoparticles are finding widespread application. There is a class of core/shell nanoparticle that has its entire constituent in the nanometer range. Core/shell nanoparticles are nano structures that have core made of a material coated with another material. They are in the size range of 8 $\mathrm{nm}$ to $40 \mathrm{~nm}$. Also, composite structures with these core/shell particles embedded in a matrix material are in use. Taking into consideration the size of the nanoparticles, the shell material can 
be chosen such that the agglomeration of particle can be prevented. This implies that the mono dispersity of the particle can be improved. The core/shell structure enhances the thermal and chemical stability of the nanoparticles, improves solubility, makes them less cytotoxic and allows conjugation of other molecules to these particles.

CHAp-CHS 20 nanocomposite particles were a bit thicker $(5-40 \mathrm{~nm})$ and longer with clear contour. In addition the particles showed less agglomeration. In addition the selected area electron diffraction (SAED, in Figure 4a) of the precipitates shows with diffraction ring of patterns, which implies that the precipitates are crystalline in nature, which is agreed by XRD results.



Figure 4. HRTEM image of CHAp-CHS 20 nanocomposite

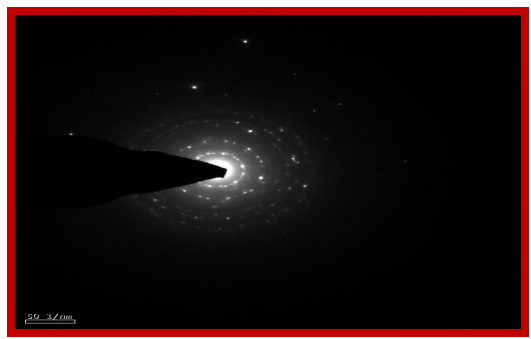

Figure 4a. SAED image of CHAp-CHS 20 nanocomposite

\section{$X$-ray photoelectron spectroscopy (XPS)}

A representative survey spectrum for HAp-CHS 20 nanocomposite powder obtained at $30{ }^{\circ} \mathrm{C}$ is presented in Figure 5 together with the expected positions of the main lines $(\mathrm{Ca} 2 \mathrm{p}$ at $347.10 \mathrm{eV}$, $\mathrm{O} 1 \mathrm{~s}$ at $531 \mathrm{eV}$ and $\mathrm{P} 2 \mathrm{p}$ at $133.8 \mathrm{eV}$ respectively. For XPS analysis the binding energies were calibrated with reference to $\mathrm{C} 1 \mathrm{~s}$ at $285 \mathrm{eV}$. The $\mathrm{Ca}(2 \mathrm{p})$ signal is composed of two lines $\mathrm{Ca} 2 \mathrm{p} 1 / 2$ $(350.67 \mathrm{eV})$ dedicated to calcium apatite and $\mathrm{Ca} 2 \mathrm{p} \mathrm{3/2}(347.13 \mathrm{eV})$ relate to a simple $\mathrm{Ca}-\mathrm{O}$ bond. As a general observation the ratio $\mathrm{Ca} / \mathrm{P}$ is nearly stoichiometric.

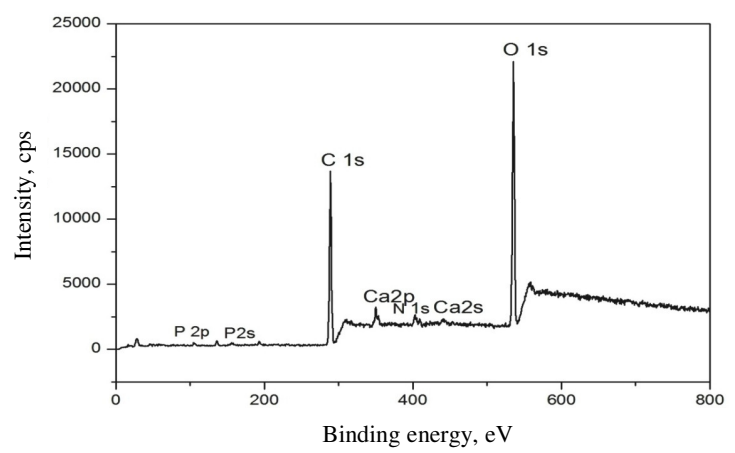

Figure 5. X-Ray photoelectron (survey) spectrum of CHAp-CHS 20 nanocomposite

\section{Electrochemical studies}

\section{Detection of 4-nitrophenol}

Cyclic voltammograms (CVs) were recorded to evaluate the electrocatalytic behaviors of the modified electrode towards the oxidation of 4-NP (4-nitrophenol) in the potential range of 0 to $+1.0 \mathrm{~V}$. Figure 6 depicts the CV's of bare, nano CHAp, nanocomposites of CHAp-CHS 20, CHAp-CHS 40, CHAp-CHS 60 and CHAp-CHS 80 modified GCE respectively in the presence of $0.5 \mathrm{mM}$ 4-NP in 0.1 M PBS(Phosphate Buffer solution). The CV of 4-NP (Figure 1) 
(Figure 6) at bare GCE shows a very broad peak at about $+0.92 \mathrm{~V}$. The 4-NP voltammogram obtained at CHAp modified GCE showed an oxidation potential at $+0.75 \mathrm{~V}$ (Figure 6). However, the 4-NP voltammogram obtained for nanocomposites of CHAp-CHS 20, CHAp-CHS 40, CHAp-CHS 60 and CHAp-CHS 80 modified GCE showed well defined oxidation wave of 4-NP with cathodic shift in potential and variation of the oxidation peak current correspond to the bare and nano CHAp modified GCE, indicating the electrocatalytic ability of the modified GCE. ${ }^{21-25}$ From the Figure 6, it can be seen that the oxidation potential and current response of 4-NP varied with the amount of the added CHS. Especially CHAp-CHS 80 modified GCE showed higher oxidation peak current with lower oxidation potential at $+0.73 \mathrm{~V}$. The overpotential had thus decreased by $0.19 \mathrm{~V}$ indicating the strong electrocatalytic effect of CHAp-CHS 80 modified layer.

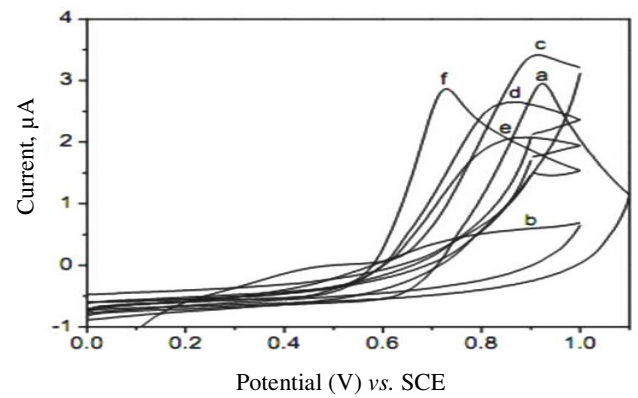

Figure 6. CV of $0.5 \mathrm{mM}$ 4-NP at (a) bare, (b) nano CHAp, (c) CHAp CHS 20, (d) CHApCHS 40, (e) CHAp-CHS 60 and (f) CHAp-CHS 80 modified GCE in the scan rate of $10 \mathrm{mV} \mathrm{s}^{-1}$

The above results indicate that catalytic reaction occurred between the CHAp-CHS 80 with 4-NP. The catalytic reaction facilitates electron transfer between NPs and the modified electrode, as a result the electrochemical oxidation of NPs becomes easier. The reason for this is that the CHAp-CHS 80 can act as a promoter to increase the rate of electron transfer, lower the overpotential of NPs at the bare electrode and the anodic peak shifts less positive potential. Thus, it is clear that CHAp-CHS 80 modified GCE can be successfully used for the determination of organic pollutant.

\section{Conclusion}

The conclusions drawn from this work are the FTIR and XRD analysis of the CHAp-CHS nano composites showed that both the components exist in the composite. The XRD pattern shows diffraction peaks with line broadening and high intensities, which confirms the nanosize with crystalline nature. HRTEM photograph of CHAp-CHS nanocomposite showed that the particles are in nanometer scale $(10-40 \mathrm{~nm})$. In addition, the particles showed less agglomeration. The selected area electron diffraction (SAED) studies showed that diffraction ring of patterns which implies that the ceramic particles are crystalline in nature, which is also proved by XRD results. The CHAp-CHS nanocomposites modified GCE showed good electrocatalytic activity towards the detection of 4-NP. The oxidation peak potentials for the nitrophenols are shifted to lower anodic region for the modified electrodes compared to bare GCE. This study will be useful for the detection of environmental organic pollutants and also traces of explosives.

\section{References}

1. Niazi A and Yazdanipour A A, J Hazard Mater., 2007, 146(1-2), 421-427.

2. Nistor C, Oubina A, Marco M P, Barceló D and Emnéus J, Anal Chim Acta, 2001, 426, 185-195. 
3. Thompson M J, Ballinger L N, Cross S E and Roberts M S, J Chromatogr B, 1996, 677, 117-122.

4. Galeano-Diaz T, Guiberteau-Cabanillas A, Mora-Diez N, Parrilla-Vazquez P and Salinas-Lopez F, J Agric Food Chem., 2000, 48(10), 4508-4513.

5. Guo X, Wang Z and Zhou S, Talanta. 2004, 64, 135-139.

6. Yang X, Shen G and Yu R, Microchim Acta, 2001, 136, 73.

7. Huang W, Yang C and Zhang de S, Anal Bioanal Chem., 375, 703.

8. Yang C H, Microchim Acta, 2004, 148, 87-92.

9. Liu Z, Du J, Qiu C, Huang L, Ma H, Shen D and Ding Y, Electrochem Commun 2009, 11, 1365

10. Kafi A K M and Chen A, Talanta, 2009, 79(1), 97-102.

11. Del Mar Cordero-Rando M, Barea-Zamora M, Barberá-Salvador J M, NaranjoRodríguez I, Muñoz-Leyva J A and Hidalgo-Hidalgo de Cisneros J L, Microchim Acta, 1999, 132, 7.

12. Hu S, Xu C, Wang G and Cui D, Talanta, 2001, 54(1), 115-123.

13. El Mhammedi M A, Achak M, Bakasse M and Chtaini A, J Hazard Mater., 2009, 163(1), 323-328.

14. Lin K, Pan J, Chen Y, Cheng R and Xu X, J Hazard Mater., 2009, 161(1), 231-240.

15. Wang B, Zhang J J, Pan Z Y, Tao X Q and Wang H S, Biosens Bioelectron., 2009, 24(5), 1141-1145.

16. El Mhammedi M A, Achak M and Chtaini A, J Hazard Mater., 2009, 161, 55-61.

17. Kong L, Gao Y, Lu G, Gong Y, Zhao N and Zhang X, Eur Polym J., 2006, 42, 3171-3179.

18. El Mhammedi M A, Bakasse M and Chtaini A, Electroanalysis, 2007, 19(16), 1727-1733.

19. Killoy W J, Int Dent J., 1998, 48(3), 305-315.

20. Raynaud S, Champion E and Bernache-Assollant D, Biomaterials, 2002, 23, 1073-1080.

21. Fischer J, Vanourkova L, Danhel A, Vyskocil V, Cizek K, Barek J, Peckova K, Yosypchuk B and Navratil T, Int J Electrochem Sci., 2007, 2, 226-234.

22. Choudhury A, Bhowmick A K and Ong C, Polymer, 2009, 50, 201-210.

23. Huanshun Yin, Yunlei Zhou, Shiyun Ai, Xianggang Liu, Lusheng Zhu and Linan Lu. Microchim Acta, 2010, 169(1-2), 87-92.

24. Dhanalakshmi C P, Vijayalakshmi L and Narayanan V, Int J Nano Mater Sci., 2012, 1(2), 81-96.

25. Niaz A, Fischer J, Barek J and Yosypchuk B, Electroanalysis, 2009, 21, 1786-1791. 